\section{Intrakranielle Gefäßmissbildungen}

Andreas Hartmann

Neurologische Klinik, Stroke Unit, Charité Campus Benjamin Franklin

psychoneuro 2005; 31 (5): 264-268

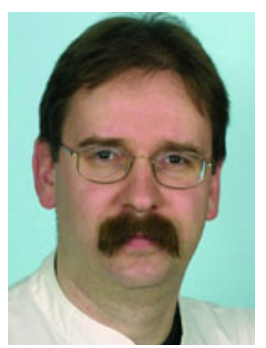

Andreas Hartmann
Intrakranielle arteriovenöse Malformationen (AVMs) werden mit moderner Bildgebung zunehmend detektiert. Klinisch manifestieren sie sich mit Blutungen und epileptischen Anfällen, seltener durch Kopfschmerzen oder neurologische Defizite. Therapeutische Optionen beinhalten die neurochirurgische Entfernung, die endovaskuläre Embolisation, die Strahlentherapie oder eine Kombination der verschiedenen Verfahren. Die prinzipielle Entscheidung zur Therapie sollte interdisziplinär fallen und stellt eine komplexe Nutzen-Risiko-Abwägung dar, die von Klinik, Lage, Größe und Angioarchitektur der AVM bestimmt wird. Andere Entitäten mit unterschiedlicher Ätiologie, Risikoprofil und therapeutischen Optionen sind V. Galeni Malformationen, durale Fisteln, kavernöse Malformationen, venöse Anomalien und kapilläre Teleangiektasien.
$\mathrm{D}$ urch den weit verbreiteten Einsatz sensitiver bildgebender Verfahren des Neurokraniums werden intrakranielle vaskuläre Malformationen mit zunehmender Häufigkeit entdeckt. Oft werfen sie Fragen nach Risiken für den betroffenen Patienten, nach Behandlungsbedürftigkeit und therapeutischen Möglichkeiten auf. Für viele dieser unterschiedlichen Entitäten sind gesicherte Daten jedoch nur spärlich vorhanden. Im Folgenden soll ein Überblick gegeben werden über die am häufigsten vorkommenden Läsionen, ihre morphologischen Charakteristika, ihre klinische Relevanz und ihre Behandlungsmöglichkeiten. Da die intrakraniellen arteriovenösen Malformationen die Gruppe mit der größten klinischen Bedeutung darstellen, sollen sie an erster Stelle und am ausführlichsten diskutiert werden

\section{Arteriovenöse Malformationen Ätiologie}

Intrakranielle arteriovenöse Malformationen (AVM) stellen anatomisch ein komplexes Konglomerat abnormer Arterien und Venen dar. Charakteristisch sind multiple Fisteln, die - bei gleichzeitig fehlenden Kapillaren - Arterien und Venen miteinander verbinden. Theorien zur Genese von AVMs reichen von embryonalen Entwicklungsstörungen über fehlerhafte Kapillarproliferationen und primäre Endotheldefekte bis zu angiogenetischen Entwicklungsstörungen. Es wird angenommen, dass die initiale zur AVM führende Läsion nach der 29. embryonalen Gestationswoche bzw. in der frühen postpartalen Phase entsteht. Insbesondere die vermehrte Expression angiogenetischer Faktoren wie z.B. VEGF (vascular endothe- lial growth factor), FGF (fibroblast growth factor), TGF (transforming growth factor), Endoglin und Angiopoietin Rezeptoren (Tie-1 und Tie-2) in AVMs Erwachsener weist auf eine Weiterentwicklung von AVMs auch nach der Postpartalphase hin.

\section{Epidemiologie}

Epidemiologische Daten zu Inzidenzen oder gar Prävalenzen von AVMs sind rar. Es ist auch letztlich nicht geklärt, welcher Anteil von AVM-Trägern symptomatisch wird. Somit bleibt eine Unsicherheit beim Rückschluss von Detektionsraten auf Prävalenzdaten, deren Größenordnung bestenfalls geschätzt werden kann. Frühe, auf Autopsieserien und retrospektiven Studien beruhende Arbeiten schätzen den Anteil von AVMs in unselektierten Populationen auf $1 \%$ bis 3\%. Modernere Studien beschreiben eine jährliche Detektionsrate von 1,0-1,4/100 000 Einwohner und schätzen eine Prävalenz von 5-100/100 000 Einwohner, allerdings bei letzterer mit großen Konfidenzintervallen $(1,3)$.

\section{Morphologie}

AVMs werden in jeder Hirnregion ohne besondere Prädilektionsstellen angetroffen, wobei Lokalisationen im Hirnstamm, Corpus callosum und in den Versorgungsgebie- 
ten der Choroidalarterien eher seltener zu finden sind. Alle an einer AVM beteiligten Einzelkomponenten, nämlich die zuführenden Arterien (sog. feeder), der Nidus und die drainierenden venösen Gefäße (Abb. 1) tragen zur morphologischen Charakterisierung und zur Prognose des Spontanverlaufs und möglicher Behandlungsrisiken bei.

Arterien: Zuführende Arterien können einzeln oder multipel vorkommen, stammen aus pialen Arterien, Grenzzonenarterien oder tiefen perforierenden Arterien. Allen ist gemeinsam, dass sie in der Regel zunächst durch gesundes Hirngewebe ziehen, dieses versorgen und dann in mehr oder weniger direktem Weg in die AVM münden. Bei häufig alterierter Muscularis und Elastica könne die feeder erheblich dilatiert sein, auch begleitende Aneurysmen werden, je nach untersuchter Serie, in 3\% bis 58\% angetroffen. Sie werden unterteilt in flussabhängige Aneurysmen auf den feedern, intranidale und nicht AVM-bezogene Aneurysmen auf entfernten Arterien.

Nidus: Im Nidus befindet sich kein normales Hirngewebe, sondern in der Regel dysplastische arterielle und venöse Gefäße. Der Nidus kann kompakt (Abb. 1) oder diffus über eine größere Hirnregion verteilt sein. Auch Malformationen mit mehreren Nidi werden angetroffen. Die Niduserkennung ist wichtig, da die meisten üblichen AVM-Klassifikationen den Nidusdurchmesser beinhalten. Der unbehandelte Nidus bleibt zwar meist größenkonstant, jedoch wurden auch Wachstum und seltener spontane Regressionen beobachtet.

\section{Venöse Drainage}

Die drainierenden Venen sind typischerweise aufgrund des hohen Shuntvolumens dilatiert. Die venösen Drainagewege folgen am häufigsten einem oberflächlichen Verlauf (Abb. 1) und münden in die großen Sinus. Alternativ werden auch die tiefen Drainagen zu den ependymalen Venen des Ventrikelsystems oder in die inneren Hirnvenen benutzt. Häufig kommen Kombinationen aus oberflächlicher und tiefer Drainage vor. In den oft ausge- dehnten venösen Kollateralgefäßen werden venöse Aneurysmen, Stenosen, Knickbildungen und Thrombosen gefunden.

\section{Klassifikation}

Es gibt viele gebräuchliche AVMKlassifikationen, die mit unterschiedlicher Gewichtung auf morphologischen, topografischen, hämodynamischen und behandlungsrelevanten Kriterien beruhen. Für die Einschätzung der operativen Komplikationsrate von AVM ist die Graduierung nach Spetzler und Martin (Tab. 1) allgemein gebräuchlich (14). Mit ansteigender Punktzahl nehmen perioperative Morbidität und Mortalität zu. Weitere behandlungs- und zugleich morphologieorientierte Klassifikationen unterscheiden wesentliche Unterformen (2):

- kleine Oberflächen-AVMs mit einer geringen Anzahl von AV-Fisteln und oberflächlicher venöser Drainage

- kortiko-subkortikale pyramidenförmige AVMs, deren Basis an der Hirnoberfläche liegt und deren Spitze oft bis zu den Ventrikeln reicht. Ihre venöse Drainage ist meist oberflächlich und tief

- tiefliegende AVMs ohne Kortexassoziation, meist in den Stammganglien, im Thalamus oder Hirnstamm

- AVMs mit starker proliferativer Komponente ohne deutlich erkennbare AV-Fisteln.

\section{Klinische Präsentation}

Die Mehrzahl der AVMs manifestiert sich typischerweise bis zum 40 . Lebensjahr, wobei Erstdetektionen auch bis zur 6. und 7. Lebensdekade beschrieben werden. Männer und Frauen sind gleicher Maßen betroffen. Die Diagnosestellung ist im CCT bei größeren AVMs meist mit Kontrastmittelunterstützung möglich, die MRT-Untersuchung detektiert AVMs sehr zuverlässig. Der Goldstandard der Diagnostik bleibt die zerebrale Angiografie, die auch Detailinformation über die Angioarchitektur der Malformation liefert.

Intrakranielle Blutungen sind mit 40\% (30-82\%) die häufigste und klinisch gefürchtetste Präsentations-

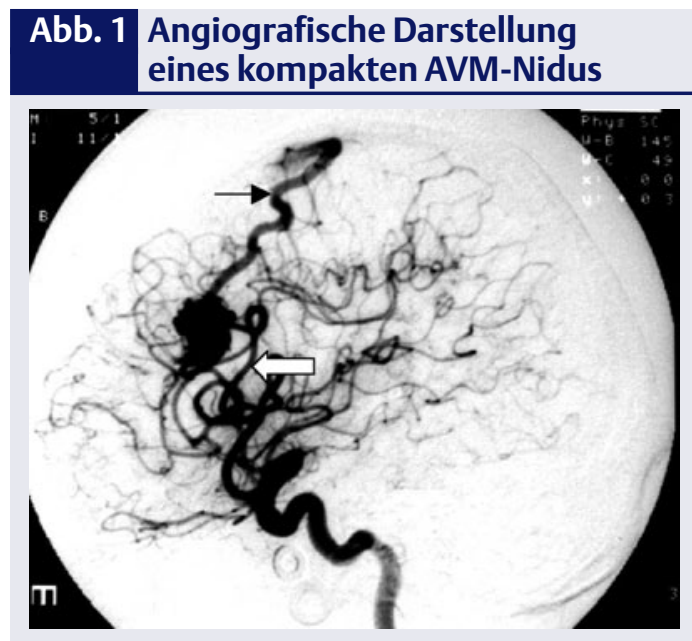

Nidus mit zuführenden Arterien aus der A. cerebri media (weißer Pfeil) und einer oberflächlich drainierenden Vene (schwarzer Pfeil)

form. Die AVM-Blutung kann auf die Region der Malformation begrenzt bleiben, sich intraparenchymatös, intraventrikulär oder subarachnoidal ausdehnen. Die durch AVMs verursachten Subarachnoidalblutungen (SABs) unterscheiden sich von den klassischen SABs, die durch die Ruptur eines Aneurysmas an den Arterien des Circulus Willisii entstehen. Bei letzteren findet sich meist eine erheblich größere Menge Blut in den basalen Liqorräumen. Ernste klinische Komplikationen wie frühe Nachblutungen, Vasospasmen, Hyponatiämien, epileptische Anfälle, EKGVeränderungen und Hydrocephalusentwicklung sind hier ebenfalls häu-

\section{Tab. 1 Spetzler-Martin-Skala}

\begin{tabular}{|c|c|}
\hline Variable & Anzahl Punkte \\
\hline \multicolumn{2}{|l|}{ AVM-Größe } \\
\hline - klein $(<3 \mathrm{~cm})$ & 1 \\
\hline - mittel $(3-6 \mathrm{~cm})$ & 2 \\
\hline - $\operatorname{groß}(>6 \mathrm{~cm})$ & 3 \\
\hline \multicolumn{2}{|l|}{ AVM-Lokalisation } \\
\hline - nicht eloquente Hirnregion & 0 \\
\hline - eloquente Hirnregion* & 1 \\
\hline \multicolumn{2}{|l|}{ Venöse Drainage } \\
\hline - nur oberflächlich & 0 \\
\hline - Beteiligung innerer Hirnvenen & 1 \\
\hline
\end{tabular}

Die summierten Punkte ergeben einen Score, der für die prognostische Einschätzung perioperativer Komplikationen von AVMs entwickelt wurde. Die AVM-Größe hat dabei den stärksten Effekt 


\begin{tabular}{lll} 
Tab.2 & \multicolumn{3}{l}{$\begin{array}{l}\text { Faktoren mit einem erhöhten } \\
\text { AVM-Blutungsrisiko }\end{array}$} \\
\hline & $\begin{array}{l}\text { Hazard } \\
\text { Ratio }\end{array}$ & $\begin{array}{l}\text { 95\% Konfidenz- } \\
\text { intervall }\end{array}$ \\
\hline Frühere Blutung & 5,38 & $2,64-10,96$ \\
\hline Assoziierte Aneurysmen & 2,70 & $1,72-4,24$ \\
\hline "Tiefe“AVM-Lage & 2,14 & $1,09-4,22$ \\
\hline Tiefe venöse Drainage & 1,84 & $1,11-3,07$ \\
\hline $\begin{array}{l}\text { Druck in feeder Arterie } \\
\text { (pro 10 } \mathrm{mmHg} \text { ) }\end{array}$ & 1,40 & $1,10-1,80$ \\
\hline AVM-Größe (pro mm) & 0,97 & $0,96-0,98$ \\
\hline AVM in Grenzzone & 0,40 & $0,26-0,60$ \\
\hline
\end{tabular}

figer. Die klassische SAB birgt ein äußerst hohes Mortalitäts- und Morbiditätsrisiko, und die Behandlung erfolgt ebenfalls im Gegensatz zu AVMs entweder durch neurochirurgische Behandlung („clipping“) oder in jüngerer Zeit zunehmend durch endovaskuläre Intervention mit Einbringen von Platinspiralen in das Aneurysma ("coiling“). Aussagen über das Blutungsrisiko von AVMs sind unsicher aufgrund mangelnder großer Längsstudien zu dem Thema. Frühere Aussagen bezifferten die jährlichen Rate von Hämorrhagien als Erstmanifestation auf 2\% bis $4 \%$ (9). Neuere Arbeiten differenzieren zwischen Blutungsraten für bislang ungeblutete AVMs von $0,1 \%$ bis $2 \%$ pro Jahr und Raten für AVMs, die bereits geblutet haben, von $1,5 \%$ bis $4 \%$ pro Jahr (8). In den ersten Jahren nach inzidenter Blutung kann das Risiko höher sein (7). In Tabelle 2 sind mehrere Faktoren aufgezählt, die das Blutungsrisiko einer AVM erhöhen können. Der klinische Schweregrad der Blutung wird bestimmt durch die Lage und die Größe der Blutung. In manchen Patientenserien gibt es Hinweise darauf, dass die Folgen einer AVM-assoziierten Blutung klinisch weniger ausgeprägt sind als Blutungen anderer, z.B. hypertensiver Genese (4).

Epileptische Anfälle ohne zugrundeliegende Blutung sind mit $30 \%(16 \%-53 \%)$ die zweithäufigste Präsentationsform von AVMs. In der Mehrzahl treten fokale oder komplex-fokale Anfälle auf, während Grand mal Anfälle in $27 \%$ bis $35 \%$ beobachtet werden. Generell ist ein gutes Ansprechen auf übliche Antikonvulsiva zu erreichen.
Blutungsunabhängiger Kopfschmerz als initiale Präsentationsform wurde bei $10 \%$ ( $7 \%$ bis $48 \%$ ) beschrieben. Hierbei ist zu berücksichtigen, dass in diesen Fällen zwar wegen Kopfschmerzen eine zerebrale Bildgebung erfolgte, die dann eine AVM zeigte, jedoch ist der kausale Zusammenhang oft unsicher. Ein AVM-typischer Kopfschmerz kann nicht postuliert werden. Auch typische migränöse Kopfschmerzen treten bei AVM-Patienten nicht häufiger auf als in der restlichen Bevölkerung.

Neurologische Defizite ohne Hinweise für eine zugrundeliegende Blutung führen nur in ca. $5 \%$ (1-40\%) aller AVM-Patienten zur Diagnose. Die weite Spannbreite reflektiert im Wesentlichen uneinheitliche Definitionen bei der systematischen Datensammlung. Nur sehr wenige Patienten weisen progrediente neurologische Defizite auf. In früheren Arbeiten als Hypotension in normales Hirngewebe versorgenden Arterien neben der AVM hypothetisiert, konnte ein solcher „steal“ bislang nicht schlüssig belegt werden. Andere Ursachen für neurologische Defizite bei diesen Patienten können in stark erweiterten Venen mit Druck auf umliegendes Hirngewebe oder Behinderungen der Liquorpassage liegen.

\section{Therapie}

Während epileptische Anfälle und Kopfschmerzen meist mit einer konservativen Therapie beherrschbar sind, ist das vorrangige Ziel einer invasiven AVM-Behandlung die Verhinderung einer intrakraniellen Blutung. Zur Behandlung stehen verschiedene Verfahren zur Verfügung.

\section{Neurochirurgische Exstirpation}

Die operative AVM-Entfernung ist die am längsten praktizierte Behandlungsmethode und sollte wegen der Komplexität der erforderlichen Technik nur in Zentren durchgeführt werden, die über Erfahrung mit AVM-Chirurgie verfügen. Vorbereitend muss eine detaillierte superselektive Angiografie vorliegen. Für die bestmögliche Schonung gesunden Hirngewebes werden funktionelles MRT, intraoperatives elektro- physiologisches mapping und moderne Navigationssysteme verwendet. Die chirurgische Behandlung hat den Vorteil, dass die AVM in der Regel komplett entfernt werden kann und somit das Blutungsrisiko schnell auf ein Minimum reduziert wird. Komplikationen der Therapie, in erster Linie Blutungskomplikationen und neurologische Defizite, werden je nach untersuchter Serie in $1 \%$ bis $40 \%$ berichtet. Prädiktoren für Komplikationen sind im Wesentlichen die AVM-Größe, Lage in tiefen oder eloquenten Hirnregionen und die tiefe venöse Drainage (13).

\section{Endovaskuläre Embolisation}

Seit den 60er Jahren werden verschiedene Materialien über endovaskuläre Katheter in die feeder Gefäße injiziert, um die Obliteration von AVMs zu erreichen. Mit zunehmender Expertise, Weiterentwicklung von Kathetertechnik und Embolisaten ist eine superselektive Katheterisierung und zumindest Teilembolisation der meisten AVMs möglich. Häufig sind mehrere Sitzungen nötig, um eine AVM entweder komplett zu verschließen, was in ca. einem Drittel der Patienten gelingt, oder die AVM so zu verkleinern, dass eine chirurgische oder strahlentherapeutischen Behandlung mit reduziertem Risiko angeschlossen werden kann. Üblicherweise benutzte Embolisate sind N-Butylcyanoacrylat und in jüngerer Zeit Onyx. Prädiktoren für Behandlungskomplikationen, in erster Linie Blutungen durch akzidentelles Verkleben der venösen Drainagewege oder seltener Defizite durch Embolisation gesundes Hirngewebe versorgender Arterien, sind zunehmende Anzahl von Embolisationen, Anzahl und Stenosen drainierender Venen, tiefe feeder Arterien und sog. high-flow shunts $(2,5)$.

\section{Strahlentherapie}

Seit Mitte der 70er Jahre wird hochdosierte, fokussierte, meist in einer Sitzung durchgeführte Bestrahlung zur Therapie von AVMs angewendet. Heutzutage wird meist mit hochfokussierten Linearbeschleunigern oder dem sog. Gamma knife gearbeitet. Dabei wird in Stereotaxietechnik eine vaskuläre Lä- 
sion in der AVM ausgelöst, die subsequent zu einer langsamen Obliteration der AVM führt (10). Dieser Prozess kann bis zu mehreren Jahren dauern. Die Blutungsneigung von AVMs in dieser Zeit und die Rate von lokalen Strahlenspätschäden bleibt unklar. Die Attraktivität der Methode liegt für viele Patienten in der geringen Invasivität. Die wesentliche Begrenzung der Strahlentherapie ist in der maximalen Größe der AVM von 30-40 mm Durchmesser begründet. AVM-Volumen $>10 \mathrm{ml}$, ein diffuser Nidus und Lage in einer eloquenten Region sind die morphologischen Prädiktoren für therapiebezogene Komplikationen.

\section{Konservative Behandlung}

Diese, auf symptomatische Behandlung ausgerichtete Therapie, bleibt als Option bei Patienten, bei denen die anderen Verfahren als zu risikobehaftet erscheinen. Das inhärente Risiko der konservativen Behandlung entspricht dem Blutungsrisiko der AVM im Spontanverlauf.

Über die differenzierte Anwendung der verschiedenen therapeutischen Verfahren sollte in Abhängigkeit von der klinischen Präsentation der AVM, ihrer Lage, Größe und Angioarchitektur in einem interdisziplinären Team entschieden werden. Dabei sind in einer Nutzen-/Risiko-Abwägung die Behandlungsrisiken der komplett anzustrebenden AVM-Entfernung oder Obliteration gegenüberzustellen. Das Fehlen randomisierter Therapiestudien stellt dabei eine zusätzliche Schwierigkeit bei der Entscheidung zur invasiven Behandlung dar.

\section{Malformationen der Vena cerebri magna Galeni}

Unter diesem Begriff werden verschiedene Missbildungen im Bereich der V. Galeni subsummiert, denen die pathologische Erweiterung der Vene bzw. ihres Vorläufers mit oder ohne venösen Aneurysmen aufgrund eines pathologischen arteriovenösen Kurzschlusses gemeinsam ist. Als embryologischer Vorläufer der V. Galeni wurde die mediane Vene des Prosencephalons identifiziert. Die eigentliche V. Galeni und der Sinus rectus bilden sich erst in der 11. embryonalen
Gestationswoche aus, so dass der vaskuläre Defekt bei der klassischen V. Galeni Malformation vor diesem Zeitpunkt entstehen muss. Dafür spricht der immer häufigere sonografische Nachweis dieser Läsion in utero, der für die später entstehenden arteriovenösen Malformationen nicht gelungen ist. Deshalb manifestieren sich V. Galeni Malformationen klinisch entweder bei Neugeborenen durch eine Herzinsuffizienz, die durch das hohe intrakranielle Shuntvolumen bedingt ist, oder spätestens bei Kleinkindern durch fokale neurologische Auffälligkeiten oder einen vergrößerten Kopfumfang. Die endgültige Diagnosestellung erfordert eine konventionelle Angiografie, und die kausale Behandlung erfolgt in der Regel durch eine frühzeitige endovaskuläre Therapie (6).

\section{- Durale arteriovenöse Fisteln}

Die meisten duralen arteriovenösen Fisteln werden aus Ästen der A. carotis externa oder aus muskelversorgenden Endästen der A. vertebralis gespeist und münden in der Mehrzahl in einen der großen intrakraniellen Sinus. Sie können angeboren sein, atraumatisch im späteren Leben z.B. im Gefolge einer intrakraniellen venösen Thrombose erworben werden oder auch als Folge eines direkten Traumas entstehen. Klinisch manifestieren sie sich in der Regel durch ein pulssynchrones Strömungsgeräusch, seltener durch Kopfschmerzen oder Zeichen des erhöhten Hirndrucks. Auch als Ursache intrakranieller Blutungen kommen sie in Frage und sollten deswegen einer Behandlung unterzogen werden. Obwohl sie in der MR-Tomografie und der MR-Angiografie detektiert werden können, ist die konventionelle Angiografie der diagnostische Goldstandard. Eine Sonderform der duralen arteriovenösen Fistel ist die Karotis-Cavernosus-Fistel. Sie kann sowohl spontan besonders bei älteren Menschen als auch als Folge eines Traumas, z.B. einen Schlag auf das Auge, entstehen. Bei dieser Läsion verbindet meist ein einziger arteriovenöser Shunt die A. carotis interna bei ihrer Passage durch den Sinus cavernosus mit einem Teil des Sinus. Der klassi- sche Symptomenkomplex beinhaltet eine injizierte Sklera und eine Bewegungsstörung des betroffenen Auges, sowie ein pulssynchrones Strömungsgeräusch, das bei der transorbitalen Auskultation mit dem Stethoskop häufig gehört werden kann. In besonders ausgeprägten Fällen kann ein Visusverlust hinzukommen. Die Behandlung kann je nach Ausdehnung und Lokalisation der Fisteln chirurgisch oder endovaskulär mittels Embolisation präferentiell von der arteriellen Seite, selten auch mittels coiling von der venösen Seite erfolgen (6).

\section{Kavernöse Malformationen}

Kavernöse Malformationen (Kavernome), die einzigen eigentlichen venösen Malformationen des $\mathrm{Ge}$ hirns, werden wegen ihrer charakteristischen Morphologie in der Kernspintomografie zunehmend häufiger diagnostiziert. Die meist rundlichen Strukturen nehmen typischerweise relativ homogen Kontrastmittel auf und weisen in den T2- oder T2*-Wichtungen häufig Hämosiderin als Zeichen stattgehabter Blutungen auf, die oft klinisch asymptomatisch bleiben. Im CCT sind besonders kleine Kavernome nur sicher $\mathrm{zu}$ detektieren, wenn sie verkalkt sind. Obwohl es sich bei Kavernomen um Raumforderungen handelt, sind sie in der Regel zu klein, um signifikante Verdrängungseffekte zu bewirken. Sie können jedoch in klinisch wichtigen kortikalen oder subkortikalen Regionen oder in der hinteren Schädelgrube lokalisiert sein. Kavernöse Malformationen bestehen aus erweiterten kavernösen, endothelialisierten Gefäßkanälen, die durch dünne Bindegewebssepten getrennt sind. Im Gegensatz zu den arteriovenösen Malformationen ist der Blutfluss durch die Kavernome nur sehr gering, weswegen sie sich der Diagnosestellung in der Angiografie entziehen. Obwohl die Malformation per se meist keine Proliferationstendenz hat, kann es durch Konfluenz benachbarter Gefäßräume oder durch rekurrente, meist lokal begrenzte Hämatome zu einer langsamen Größenzunahme kommen. Das klinische Manifestations- 
spektrum von Kavernomblutungen reicht von asymptomatisch bis zu schwersten Defiziten bei Blutungen in den Hirnstamm oder andere eloquente Hirnregionen. Selten werden sie als Fokus von epileptischen Anfällen entdeckt. Multiple Kavernome kommen gelegentlich vor und auch familiäre Häufungen mit autosomal dominantem Erbgang wurden beschrieben. Als Genloci wurden Veränderungen auf den Chromosomen 3 und 7 entdeckt. Ob kavernöse Malformationen behandelt werden müssen, ist meist eine Einzelfallentscheidung. $\mathrm{Zu}$ einer Therapie wird man sich in der Regel entscheiden, wenn eine klinisch manifeste Blutung stattgefunden hat oder bildmorphologisch im Verlauf eine Größenzunahme oder rekurrente Blutungen dokumentiert werden. Als therapeutisches Verfahren steht die neurochirurgische Entfernung zur Verfügung. In jüngerer Zeit wird auch über Erfahrungen mit der stereotaktischen Bestrahlung berichtet, wobei die Datenlage hierzu noch dünn ist.

\section{Venöse Fehlbildungen}

Im angloamerikanischen Sprachraum werden diese häufig vorkommenden Gefäße auch als „developmental venous anomalies (DVAs)“ bezeichnet, da sie nicht als Malformationen im engeren Sinne, sondern eher als extreme anatomische Varianten der venösen Drainage des Gehirns einzuschätzen sind. Sie werden oft in der MR-Tomografie diagnostiziert und lassen sich in den Spätphasen konventioneller Angiografien in typischer Weise ohne arteriellen Zufluss bestätigen. Sie bestehen aus fingerförmig zusammenlaufenden kleinen Venen („Medusenhaupt“), die in eine große, oft transmedullär verlaufende Vene münden. Eine Assoziation mit kavernösen Malformationen wurde in bis zu 30\% beschrieben (12). Klinisch bleiben sie meist asymptomatisch. Ihre Beteiligung an intrakraniellen Blutungen ist umstritten und nur im Fall einer venösen Abflussstörung, etwa im Rahmen einer Thrombose, zu diskutieren (3). Eine Behandlung ist in der Regel nicht erforderlich.

\section{- Kapilläre Teleangiektasien}

Hierbei handelt es sich um meist asymptomatisch bleibende kleine Anhäufungen kapillar-artiger Gefäße ohne arteriovenöse shunts, die oft im Hirnstamm oder im Kleinhirn zu finden sind. Früher in der Regel zufällig bei Autopsien beobachtet, können sie im MRT als kleine, streifig aussehende Läsionen erhöhter Signalintensität diagnostiziert werden. Ihre klinische Relevanz ist zweifelhaft. Obwohl manchmal morphologische Zeichen stattgehabter Mikroblutungen zu finden sind, wurden sie nur sehr selten in Verbindung mit symptomatischen Hirnblutungen gefunden. Eine invasive Behandlung ist normalerweise nicht indiziert. Eine genetisch determinierte Form mit autosomal dominantem Erbgang (11) und mittlerweile auf Chromosom 12 lokalisiertem Genlokus wurde als hereditäre hämorrhagische Teleangiektasie klassifiziert (M. Rendu-Osler-Weber). Bei diesen Patienten findet man sie häufig an multiplen Stellen im Gehirn, in 3\% assoziiert mit z.T. mehreren arteriovenösen Malformationen, sowie in anderen Organen.

Intracranial vascular malformations Modern imaging techniques lead to increasing diagnoses of intracranial arteriovenous malformations (AVMs). They present clinically with intracranial hemorrhage and seizures, less frequently with headache or neurological deficits. Therapeutic options include neurosurgical resection, endovascular obliteration, radiosurgery or a combination of the different treatment modalities. Decisions for invasive therapy should be interdisciplinary and require complex risk-benefit considerations. Mode of clinical presentation, size, location and angioarchitecture of the AVM influence treatment decisions. Other entities of different etiology, risks, and treatment incentives are Vein of Galen malformations, dural fistulae, cavernous malformations, developmental venous anomalies, and capillary telangiectasies.

\section{Key Words}

arteriovenous malformations - AVM vascular malformations - therapy

\section{Literatur}

1. Al Shahi R, Warlow C. A systematic review of the frequency and prognosis of arteriovenous malformations of the brain in adults. Brain 2001; 124: 1900-1926

2. Berenstein A, Lasjaunias P. Endovascular treatment of cerebral lesions. In: Berenstein A, Lasjaunias P (eds.). Surgical Neuroangiography. New York, Berlin, Heidelberg: Springer Verlag, 1992

3. Brown RD Jr., Wiebers DO, Torner JC, O'Fallon WM. Incidence and prevalence of intracranial vascular malformations in Olmsted County, Minnesota, 1965 to 1992. Neurology 1996; 46(4): 949-952

4. Hartmann A, Mast H, Mohr JP, Koennecke $\mathrm{HC}$, Osipov A, Pile-Spellman jet al. Morbidity of intracranial hemorrhage in patients with cerebral arteriovenous malformation. Stroke 1998; 29(5): 931-934

5. Hartmann A, Pile-Spellman J, Duong DH, Young WL, Mohr JP, Mast H. Neurologic assessment of the frequency and severity of complications following endovascular treatment of intracranial arteriovenous malformations. Cerebrovascular Diseases 1999; 9 (Suppl 1): 47

6. Lasjaunias P. In: Lasjaunias P (ed.). Vascular diseases in neonates, infants and children. Interventional neuroradiology management. Berlin: Springer-Verlag, 1997

7. Mast H, Young WL, Koennecke HC, Sciacca RR, Osipov A, Pile-Spellman J et al. Risk of spontaneous haemorrhage after diagnosis of cerebral arteriovenous malformation. Lancet 1997; 350(9084): 1065-1068

8. Mohr JP, Hartmann A, Mast H, Pile-Spellman J, Schumacher HC, Stapf C. Arteriovenous malformations and other vascular anomalies. In: Mohr JP, Choi DW, Grotta JC, Weir B, Wolf PA, editors. Stroke: Pathophysiology, Diagnosis, and Management. Philadelphia: Churchill Livingstone, 2004: 397421

9. Ondra SL, Troupp H, George ED, Schwab $\mathrm{K}$. The natural history of symptomatic arteriovenous malformations of the brain: a 24 year follow-up assessment. J Neurosurg 1990; 73(3): 387-391

10. Pollock BE. Stereotactic radiosurgery for arteriovenous malformations. Neurosurg Clin N Am 1999; 10(2): 281-290

11. Porteous ME, Burn J, Proctor SJ. Hereditary haemorrhagic telangiectasia: a clinical analysis. J Med Genet 1992; 29(8): 527-530 12. Rabinov JD. Diagnostic imaging of angiographically occult vascular malformations. Neurosurg Clin N Am 1999; 10(3): 419-432

13. Solomon RA, Stein BM. Management of arteriovenous malformations of the brain stem. J Neurosurg 1986; 64(6): 857-864

14. Spetzler RF, Martin NA. A proposed grading system for arteriovenous malformations. J Neurosurg 1986; 65(4): 476-483

Korrespondenzadresse:

PD Dr. Andreas Hartmann Neurologische Klinik, Stroke Unit Charité Campus Benjamin Franklin Hindenburgdamm 30 12200 Berlin

andreas.hartmann@charite.de 